\title{
Neue Behandlungsstrukturen in der Psychiatrie - Chance für eine zukunftsfähige Versorgung
}

\author{
Innovative Treatment in Psychiatry - A Chance for Integrated Health Care
}

Autoren

Institute
Arno Deister ${ }^{1}$, Bettina Wilms ${ }^{2}$

${ }^{1}$ Zentrum für Psychosoziale Medizin, Klinikum Itzehoe

${ }^{2}$ Klinik für Psychiatrie, Psychotherapie und Psychosomatik, Südharz-Klinikum Nordhausen

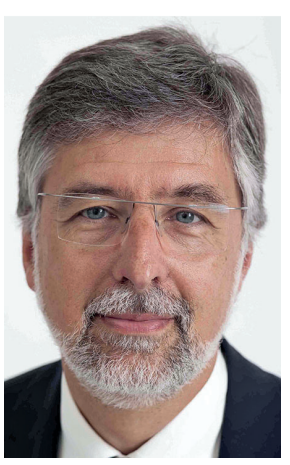

Arno Deister

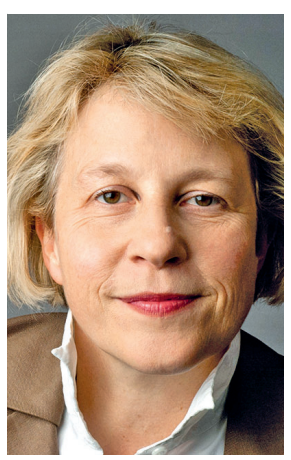

Bettina Wilms

Bibliografie

Dol http://dx.doi.org/

10.1055/s-0034-1387487

Psychiat Prax 2015; 42: 8-10

(c) Georg Thieme Verlag KG

Stuttgart · New York

ISSN 0303-4259

Korrespondenzadresse

Prof. Dr. med. Arno Deister

Chefarzt des Zentrums für

Psychosoziale Medizin

Klinikum Itzehoe

Robert-Koch-Straße 2

25524 Itzehoe

a.deister@kh-itzehoe.de
Wir haben jetzt noch eine Chance. Wir haben die Chance, das System der Hilfe und Versorgung für Menschen mit psychischen Erkrankungen zielgerichtet weiterzuentwickeln. Nutzen wir diese Chance?

Die psychiatrische und psychotherapeutische Versorgungslandschaft steht vor großen Herausforderungen. Einerseits steigt die Inanspruchnahme von Leistungen in diesen Bereichen, andererseits wird der bereits real bestehende und zukünftig eher zunehmende Mangel an dafür erforderlichen Ressourcen immer deutlicher. Seit Jahren gibt es vonseiten der Kostenträger (und der Gesundheitspolitik) Bestrebungen, die Ausgabensteigerungen zu begrenzen; gleichzeitig zeigt sich ein Mangel an qualifizierten Mitarbeiterinnen und Mitarbeitern. Hinzu kommt der viel beschriebene demografische Wandel, der zu weiteren grundlegenden Veränderungen führen wird. Ein neues und innovatives System der Finanzierung von psychiatrischen und psychotherapeutischen Leistungen ist in dieser Situation eigentlich unverzichtbar. Die Diskussion über ein neues Finanzierungssystem für die Psychiatrie und Psychotherapie wird aktuell heftig und kontrovers geführt. Zu häufig geht es dabei aber primär um formale Aspekte der Abrechnung von eher kleinteiligen Einzelleistungen oder um die Definition von sog. „Kostentrennern“. „Transparenz“ scheint das Zauberwort zu sein für „Aufwand“ und „Überprüfbarkeit“. Es steht der Vorwurf im Raum, dass Psychiatrie und Psychotherapie sonst eine qualitätsfreie Black Box seien. Das, was unsere Leistungen - unser Fach insgesamt - eigentlich ausmacht, gerät in den Hintergrund oder wird als sog. „Grundrauschen“ inhaltlich und ökonomisch abgewertet: Das therapeutische Milieu genauso wie z.B. partizipative Entscheidungsfindung oder das Aushalten von aktuell nicht zu minderndem Leiden. Tragfähige Anreize dazu, Versorgungsstrukturen und Versorgungsangebote zu entwickeln, die wirklich geeignet sind, den Bedürfnis- sen von Menschen mit psychischen Erkrankungen zu entsprechen, gibt es praktisch nicht. Der Blick richtet sich in erster Linie auf die Finanzierung von Teilprozessen statt auf die Qualität von Behandlungsergebnissen. Ein daraus entstehendes System von Einzelleistungen und Einzelleistungsvergütungen ist die logische Folge [1, 2].

Die Notwendigkeit der strukturellen Voraussetzungen für ein zukunftsfähiges Versorgungssystem - der strukturellen Qualität - wird uns erst langsam deutlich. In keinem anderen medizinischen Fachgebiet ist die Notwendigkeit einer am ganzen Menschen orientierten integrativen Versorgung deutlicher als im Gebiet der Psychiatrie und Psychotherapie. Gleichzeitig ist kein Fach besser geeignet, diese integrative Versorgung auch praktisch umzusetzen. Psychische Erkrankungen sind grundsätzlich dadurch gekennzeichnet, dass sie den ganzen Menschen und sein psychosoziales Umfeld betreffen. Menschen, die an einer psychischen Störung leiden, haben regelhaft krankheitsbedingte Einschränkungen in ihren privaten und sozialen Beziehungen. Die gleichberechtigte gesellschaftliche Teilhabe ist eine der wesentlichen Bedingungen für deren Genesung. In der Psychiatrie und der Psychotherapie haben sich in den letzten Jahren verschiedene Formen integrativer Versorgung modellhaft entwickeln und teilweise bereits etablieren können. Der §64b im Sozialgesetzbuch V bietet Möglichkeiten dazu, umfassende und regional vernetzte Modellprojekte zu entwickeln und umzusetzen. Demnach sollen diese „beitragen zu einer Weiterentwicklung der Versorgung psychisch kranker Menschen, die auf eine Verbesserung der Patientenversorgung oder der sektorenübergreifenden Leistungserbringung ausgerichtet ist, einschließlich der komplexen psychiatrischen Behandlung im häuslichen Umfeld“ (SGB V). Modellprojekte bieten damit die Möglichkeit, die überkommenen und weitgehend starren Grenzen zwischen unterschiedlichen Behandlungsformen und Behand- 
lungssettings (stationär, tagesklinisch und ambulant) zu überwinden. Dies bedeutet nicht nur eine effizientere Nutzung der vorhandenen Ressourcen, sondern vor allem die Möglichkeit der Ausrichtung der Versorgungsstrukturen an den Bedürfnissen von Menschen mit psychischen Erkrankungen. Die Grundbedingung für innovative Versorgungsstrukturen muss sein, dass sie von einem fragmentierten System der Behandlung von Krankheiten hinführen zu einer integrativen Förderung von Gesundheit, in der präventive, therapeutische, rehabilitative und manchmal auch palliative Aspekte eng aufeinander bezogen und vernetzt sind. Dies erfordert grundlegend neue Anreize und Steuerungsformen in der Region.

Was macht nun neue und innovative Versorgungsformen wirklich aus? Ein an den Bedürfnissen von Menschen mit psychischen Erkrankungen ausgerichtetes Behandlungssystem muss sich daran messen lassen, in welchem Maße es die folgenden Kriterien erfüllt [1-3]:

- Sicherstellung der erforderlichen Behandlungsqualität

- Flexibilisierung der Behandlungsmöglichkeit

- Förderung des Prinzips „ambulant statt stationär“

- Vernetzung der Angebote

- Reduktion des Drehtüreffekts

- Reduktion des Misstrauensaufwands

In gleicher Weise müssen auch fachlich gebotene Behandlungsprozesse gefördert werden. Dabei handelt es sich in erster Linie um:

- weitgehende Symptomreduktion bei gleichzeitiger Fähigkeit zur Teilhabe am Leben

- Aktivierung von Ressourcen und Förderung der Motivation

- Förderung der Eigeninitiative und Empowerment

- Vorhandensein bzw. Ausbau stabiler sozialer Kontakte

- partizipative Entscheidungsfindung

- langfristige Beziehungs- und Behandlungskonstanz

- Vermeidung von häufigen Wiederaufnahmen, die durch Brüche in der therapeutischen Kontinuität bedingt sind Die bisher in Deutschland umgesetzten modellhaften Projekte für die Versorgung von Menschen mit psychischen Erkrankungen sind weder konzipiert noch geeignet dafür, in allen Regionen in Deutschland kurzfristig und in gleicher Weise umgesetzt zu werden. Die im Rahmen der Umsetzung und der Evaluation der neuen Versorgungsformen gewonnenen Erkenntnisse und die daraus entwickelten Qualitätsstandards sind jedoch durchaus geeignet, Anforderungen an ein zukünftiges regelhaftes Versorgungs- und Finanzierungssystem zu formulieren und einzufordern.

In Deutschland gibt es aktuell in 14 Regionen in 7 Bundesländern Modellprojekte nach §64b SGB V. In diesen Regionen leben insgesamt etwa 2,5 Mill. Menschen. Diese Projekte basieren in ihrer Mehrzahl auf regionalen Budgets, die seit 2003 schrittweise umgesetzt wurden. Diese Modellprojekte wurden als verändertes Finanzierungsmodell für psychiatrische und psychotherapeutische Leistungen konzipiert, die durch das Krankenhaus erbracht werden. Nicht wie bisher tagesgleiche Pflegesätze, sondern die Anzahl der behandelten Menschen ist die Finanzierungsgrundlage für das Krankenhaus. Damit kommt es zur Entkopplung eines spezifischen Behandlungssettings von der Bezahlung durch die Kostenträger. Nicht die stationäre oder teilstationäre Behandlung bringt das Geld, sondern die Behandlung einer bestimmten Anzahl von Menschen aus der Region. Letztlich beinhaltet dies in allen Regionen, die mit dieser Form eines Capitation-Modells arbeiten, dass das bisher erlöste Geld dem Krankenhaus weiterhin zur Verfügung steht, eine Leistungsausweitung allein aus betriebswirtschaftlichen Motiven begrenzt wird und ein Anreiz ge- schaffen ist, mit den im Krankenhaus zur Verfügung stehenden Mitteln flexibler und damit effizienter umzugehen [1, 2]. Weitere Modellprojekte basieren auf bisherigen Projekten der integrierten Versorgung, die sich zum Teil auch auf die ambulante Versorgung im Bereich der niedergelassenen Ärzte beziehen.

Durch diese Organisationsformen können inhaltliche Veränderungen psychiatrisch-psychotherapeutischer Arbeit zum Zuge kommen: insbesondere aufsuchende gemeindebasierte Behandlungsformen, u.a. in der Form des therapeutischen Assertive Community Treatment (ACT) und des Hometreatment, können so flächendeckend umgesetzt werden. Multiprofessionelle gemeindepsychiatrische Teams ermöglichen ein gemeindenahes Komplexangebot für Menschen mit schwerer psychischer Erkrankung. Sie orientieren sich an den individuellen Hilfsbedürfnissen der betreuten Patienten. Ziel dieser Teams ist es, die Patienten darin zu unterstützen, ein unabhängiges Leben in ihrem Lebensumfeld zu führen. Im ACT kann eine langfristige und hochintensive Behandlung von einem multidisziplinären Team (vorwiegend) im häuslichen Umfeld erfolgen. In den Angeboten des Hometreatment wird insbesondere psychiatrische Akutbehandlung durchgeführt. Hometreatment wendet sich an Menschen mit akuten psychischen Erkrankungen, bei denen grundsätzlich die Indikation für eine akutstationäre Behandlung gegeben ist. Angestrebt wird ein multidisziplinäres Behandlungsangebot mit häuslichen Kriseninterventionen und der gleichzeitigen Berücksichtigung klinischer und psychosozialer Aspekte in einer flexiblen Kommunikation mit dem Patienten und seinem sozialen Netzwerk [2, 4]. Durch das Prinzip der bedürfnisangepassten Behandlung von Menschen mit psychischen Erkrankungen (Need Adapted Treatment) können zusätzlich auch erweiterte psychotherapeutische und psychosoziale Konzepte verwirklicht werden, die die Patientinnen und Patienten als Experten für ihre eigene Lebenssituation stärker miteinbeziehen.

Die wissenschaftliche Begleitforschung für die bereits seit Langem laufenden regionalen Psychiatriebudgets konnte zeigen, dass es im Vergleich zu Regionen mit traditioneller Versorgungsstruktur zu einer signifikanten Verbesserung des Funktionsniveaus und der sozialen Integration der Patienten gekommen ist $[5,6]$. Die Verweildauer im stationären Bereich wurde relevant vermindert und gleichzeitig der ambulante Versorgungsanteil deutlich erhöht. Die Evaluation von Maßnahmen des Hometreatment zeigt, dass die Akutbehandlung im häuslichen Umfeld das Risiko eines Behandlungsabbruchs senkt, die Behandlungszufriedenheit erhöht und kosteneffektiver im Vergleich mit herkömmlicher stationärer Behandlung sein kann $[4,7]$.

Was also können wir lernen aus Modellprojekten? Neue Versorgungsstrukturen und neue Versorgungsangebote in der Psychiatrie und Psychotherapie sind geeignet, zu therapeutischen Angeboten beizutragen, die individueller und weniger an bestehenden Strukturen ausgerichtet sind als bisher und sich damit flexibler an die besonderen Bedürfnisse von Menschen mit psychischen Erkrankungen anpassen. Die konsequente Berücksichtigung des sozialen Kontextes und Therapie im bestehenden sozialen Umfeld führt zu einer verbesserten sozialen Integration von Patienten. Voraussetzung für eine breitere Anwendung in der Routineversorgung ist die weitere konsequente Erprobung und wissenschaftliche Evaluation unterschiedlicher Ansätze in Regionen mit unterschiedlichem Versorgungsbedarf. Der Einsatz von neuen Behandlungsansätzen, die auch geeignet sein können, stationäre Behandlung zu ersetzen, benötigt allerdings zwingend darauf ausgerichtete neue Strukturen der Finanzierung. Eines aber ist schon jetzt sicher: Wir wissen, dass psychiatrische und psy- 
chotherapeutische Versorgung verbessert werden kann. Dahinter sollten wir nicht mehr zurück. Wir haben jetzt noch eine Chance - maximal 2 Jahre. Wir müssen sie im Sinne unserer Patientinnen und Patienten nutzen.

\section{Literatur}

1 Deister A, Wilms B. Regionale Verantwortung übernehmen. Modellprojekte in Psychiatrie und Psychotherapie nach §64b SGB V. Köln: Psychiatrie Verlag; 2014

2 Wilms B, Becker T, Lambert $M$ et al. Modelle für eine zukunftsfähige psychiatrische Versorgung. Psychiatrie 2012; 9: 4-13

3 Kruckenberg P, Beine K, Aderhold V. Psychiatrisch-psychotherapeutisch-psychosomatische Behandlung durch das Krankenhaus. Rahmenbedingungen zur Entwicklung eines sektorübergreifenden Bud- gets für die regionale Pflichtversorgung. Psychiat Prax 2009; 36 : $246-251$

4 Deutsche Gesellschaft für Psychiatrie und Psychotherapie, Psychosomatik und Nervenheilkunde (DGPPN). S3-Leitlinie Psychosoziale Therapien bei schweren psychischen Erkrankungen. Berlin: Springer; 2012

5 Deister A, Zeichner D, Witt T et al. Veränderungen der psychiatrischen Versorgung durch ein Regionales Psychiatriebudget. Psychiat Prax 2010; 37: 335 - 342

6 König HH, Heinrich S, Heider D et al. Das Regionale Psychiatriebudget (RPB): Ein Modell für das neue pauschalierende Entgeltsystem psychiatrischer Krankenhausleistungen. Psychiat Prax 2010; 37: 34-42

7 Lambert M, Naber D, Bock $T$ et al. Integrierte Versorgung von Patienten mit psychotischen Erkrankungen - das „Hamburger Modell“. Soziale Psychiatrie 2010; 127: 30-33 